\title{
Assessing preferential flow through an unsaturated waste rock pile using spectral analysis
}

\author{
Paolo Trinchero, ${ }^{1}$ Roger Beckie, ${ }^{2}$ Xavier Sanchez-Vila, ${ }^{3}$ and Craig Nichol ${ }^{4}$ \\ Received 27 October 2010; revised 15 April 2011; accepted 3 June 2011; published 19 July 2011.
}

[1] Waste rock piles are an outcome of open pit and underground mining operations. Unprocessed low-grade rock is disposed of in piles from several meters high to $100 \mathrm{~m}+$ high. Waste rock piles may still contain sufficient concentrations of metals to be a potential source of pollution. The evaluation of the potential risk involves properly characterizing flow through these piles under unsaturated conditions. The main flow characteristic of the piles is the presence of a large range of grain and pore sizes. Based on data from an instrumented rock pile located in Saskatchewan Canada, unsaturated flow through the pile is modeled as a linear system after separating a fast and a slow component. Water reaching the base of the pile is monitored by 16 contiguous zero-tension lysimeters. The fast component, flowing through macropores, is assumed to be released instantaneously, while the slow component is simulated using a linear-reservoir model that assumes the presence of an interconnected porous matrix. An empirical transfer function (TF) is computed as the ratio of the spectra of signals between the output (basal outflow) and the input (rainfall time series). Determination of a parametric transfer function model provides information on the characteristic time of water storage in the matrix and on the fraction of the water within each subsection of the experimental pile that is channeled through the macropores. An analysis of the output signal at different support scales is performed, indicating the nonlinearity of the macropore fraction scaling processes.

Citation: Trinchero, P., R. Beckie, X. Sanchez-Vila, and C. Nichol (2011), Assessing preferential flow through an unsaturated waste rock pile using spectral analysis, Water Resour. Res., 47, W07532, doi:10.1029/2010WR010163.

\section{Introduction}

[2] Mining is a significant economic activity and has a clear socioeconomic impact in communities worldwide. The nature of mining processes and activities also create the potential for negative environmental impacts both during the mining operations and after the mine is closed. Mines produce two dominant types of waste material: tailings which are fine particles derived from the processing plant; and coarse-sized waste rock extracted from the mine in order to access the ore grade material. Coarse waste rock is typically stored in large dumps or piles sometimes over tens and even hundreds of meters high. Dumping results in a high porosity for the waste material, with the pore sizes spanning a larger range than most natural soils, potentially leading to the formation of macropores. Dumping of such an unclassified material also results in a clear impossibility of properly characterizing the medium, adding the issue of uncertainty to that of heterogeneity.

\footnotetext{
${ }^{1}$ AMPHOS 21 Consulting S. L., Barcelona, Spain.

${ }^{2}$ Department of Earth and Ocean Sciences, University of British Columbia, Vancouver, British Columbia, Canada.

${ }^{3}$ Department of Geotechnical Engineering and Geosciences, Universitat Politecnica de Catalunya BarcelonaTech, Barcelona, Spain.

${ }^{4}$ Department of Earth and Environmental Sciences, University of British Columbia Okanagan, Kelowna, British Columbia, Canada.
}

Copyright 2011 by the American Geophysical Union. 0043-1397/11/2010WR010163
[3] Water infiltrating through the piles after rainfall events reacts with the existing minerals, changing the solution chemistry in the process. Determination of in situ geochemical reaction rates in mine waste-rock piles remains a challenge [Birkham et al., 2003]. A large number of attempts to predict chemical reactions within rock piles can be found in the literature [e.g., Sracek et al., 2004; Kabwe et al., 2005; Molson et al., 2005; da Silva et al., 2009]. Prediction of solute release to the environment is limited by the inability to accurately predict field-scale weathering rates. While the geochemistry of mine waste has received significant attention, the effect of flow on geochemistry has not, and despite recent efforts, processes controlling flow through waste rock are not yet well understood [e.g., Webb et al., 2008; Poisson et al., 2009; Reiter, 2009].

[4] The chemical composition of water released from mine waste is strongly controlled by the residence time of the water within the medium. Waste rock piles are highly heterogeneous leading to a separation of fast and slow flows. Matrix flow is the term used to describe flow in a partially saturated porous media that can be largely described using Richards' equation, which sometimes requires accounting for a degree of spatial heterogeneity or hysteresis. A recharge event at the surface will generate a wetting front (approximately the horizontal plane at which there is an increase in water content and water velocity) which propagates downward. Wetting fronts propagate faster than the true velocity of water within the pile, with measured differences being $10 \mathrm{~s}$ to $1000 \mathrm{~s}$ of times faster 
[e.g., Rasmussen et al., 2000; Nimmo, 2007]. When gravity forces dominate, the wetting front velocity can be most simply expressed by the kinematic velocity, which also equals the slope of the unsaturated hydraulic conductivity to moisture content curve when fronts are small [e.g., Smith, 1981; Yamada and Kobayashi, 1988; Rasmussen et al., 2000]. This latter formulation is also the limit reached for Philip's infiltration for small changes in water content [Philip, 1958; Jury et al., 1991]. More exact solutions accommodating both capillary and gravity forces can be derived [e.g., Rasmussen et al., 2000].

[5] In contrast to matrix flow is preferential flow, which designates when there is a flow component that is spatially concentrated, or has a significantly different residence time (true water velocity) than the majority of the other water in the system. Preferential flow is typically identified in a tracer test when the initial pulse of solute splits into a main pulse, moving slowly, and involving the largest fraction of water, and a second more quickly moving front [e.g., Roth et al., 1991; Eriksson et al., 1997]. In the last decades, numerous studies have documented the existence of preferential flow at the field scale and it should be considered the norm, rather than the exception, but varying in intensity. Mechanisms of preferential flow include: (1) macropores generated by cracks or spatial arrangement of well-structured soils [Beven and Germann, 1982], (2) earthworms burrows [Edwards et al., 1989] and root channels [Gish and Jury,

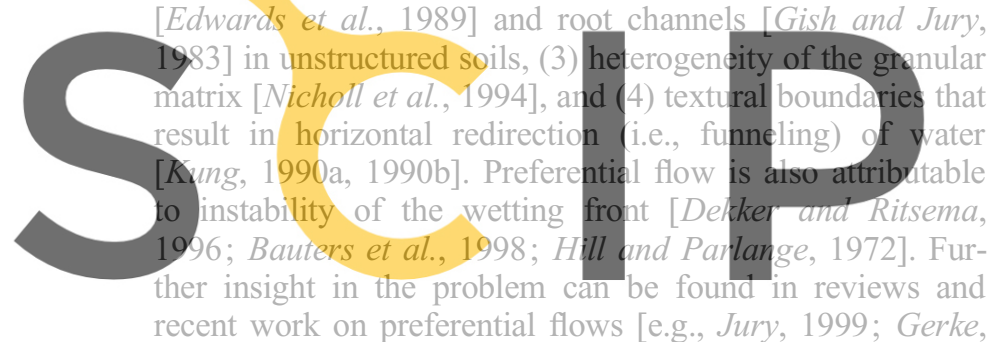

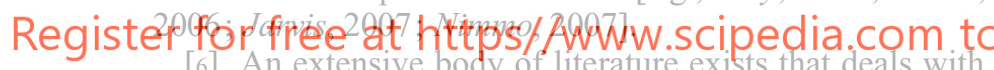
different approaches to model flow in unsaturated structured soils [Simunek et al., 2003; Gerke, 2006; Jarvis, 2007]. Starting from the more general flow equation, different alternatives have been used to simplify the flow problem, including a single domain Richards' equation, formulations with heterogeneity or hysteresis, and the development of dual-domain approaches. The latter consist in either assuming that flow and transport are restricted to fractures and macropores where the matrix is considered an immobile zone (dual-porosity models) [van Genuchten and Wierenga, 1976] or considering that the rate of flow and transport is different in each zone (dual-permeability models) [Gerke and van Genuchten, 1993]. Field experiments support the applicability of these dual models [White et al., 1986] and they have been used to describe flow in mine materials [e.g., Decker and Tyler, 1999].

[7] The difficulty of implementing mechanistic approaches such as dual porosity models arises from the need of a rigorous microscopic description of the medium to obtain reliable hydraulic parameters at the scale of interest. This description is often infeasible in real applications where the size of the medium and the high heterogeneity of its textural structure make a detailed characterization of its hydraulic properties impossible. Usually a tracer test is required to be able to parameterize these models. Recent field scale studies specific to mine waste rock [e.g., Nichol et al., 2005; Webb et al., 2008] have identified significant preferential flow behavior. The range of grain sizes in waste rock and the subsequent scales of heterogeneity complicate the use of laboratory scale tracer tests to parameterize large scale models. Field tests are not often practical due to the substantial residence times in scale-appropriate test waste rock piles. Water table fluctuation methods can be used to recover averaged flow data underneath piles [e.g., Lopez et al., 1997].

[8] Here we investigate a new method, based on flow data, to separate preferential flow from matrix flow by focusing on the average behavior of the system. The approach we pursue assumes that the medium behaves as a linear filter and simulates the output of the system (in this case, the outflow) as the convolution of an input signal with a linear filter, the latter being defined by means of a transfer function $T F$. This methodology has been extensively used in many hydrogeological studies [Jury, 1982; Jury et al., 1986; White et al., 1986] including the analysis of spring flow from karst aquifers [Dreiss, 1983; Long and Derickson, 1999; Denic-Jukic and Jukic, 2003].

[9] The linear filter approach aims at identifying the average behavior of the system and specifically the mean rate of preferential flow occurring and a characteristic time scale of the flow through the matrix. The model is implemented by computing the empirical TF of lysimeters at the

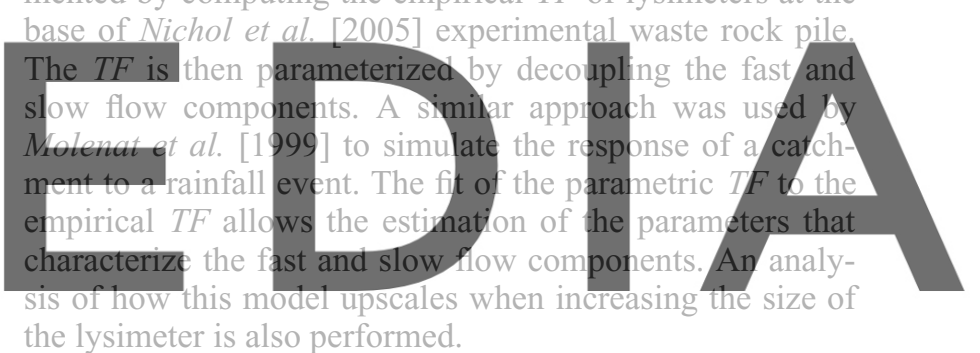

the lysimeter is also performed.

\section{download the version without the watermark}

\section{Study Area and Monitoring Scheme}

[10] The data set that we examine here comes from the waste-rock pile experiment instrumented by Nichol et al. [2005] at the Cluff Lake Uranium Mine in northern Saskatchewan (Canada). The mine rocks are composed of aluminous gneisses and granitoids from the Precambrian Peter River Gneiss formation. The waste rock at the Cluff Lake Mine has a broad grain size distribution, which includes boulders up to $1.0 \mathrm{~m}$ in diameter. The proportion of fine material $(<2 \mathrm{~mm}$ ) largely falls at the boundary of piles considered rock-like where there are insufficient fines $(<20 \%)$ to fill the gravel to boulder interstices, or soil-like $(>20 \%)$ where larger particles are generally surrounded by fine-grained material [Smith and Beckie, 2003].

[11] An instrumented experimental waste-rock pile was constructed at the site in 1998 and was designed to mimic the behavior of the upper meters of a much larger unsaturated rock pile. Simplified plan and cross sectional views of the pile are shown in Figure 1. The instrumented core of the pile had a footprint of $8 \mathrm{~m}$ by $8 \mathrm{~m}$ and is $5 \mathrm{~m}$ high. Outflow from the base of the pile was collected in a contiguous grid of 16 lysimeters (Figure 1, inset). The pile rested on a contoured cement pad lined with a PVC geomembrane. Plywood lined with a 60 mil HDPE geomembrane was placed from the base of the pile to the surface to isolate the 

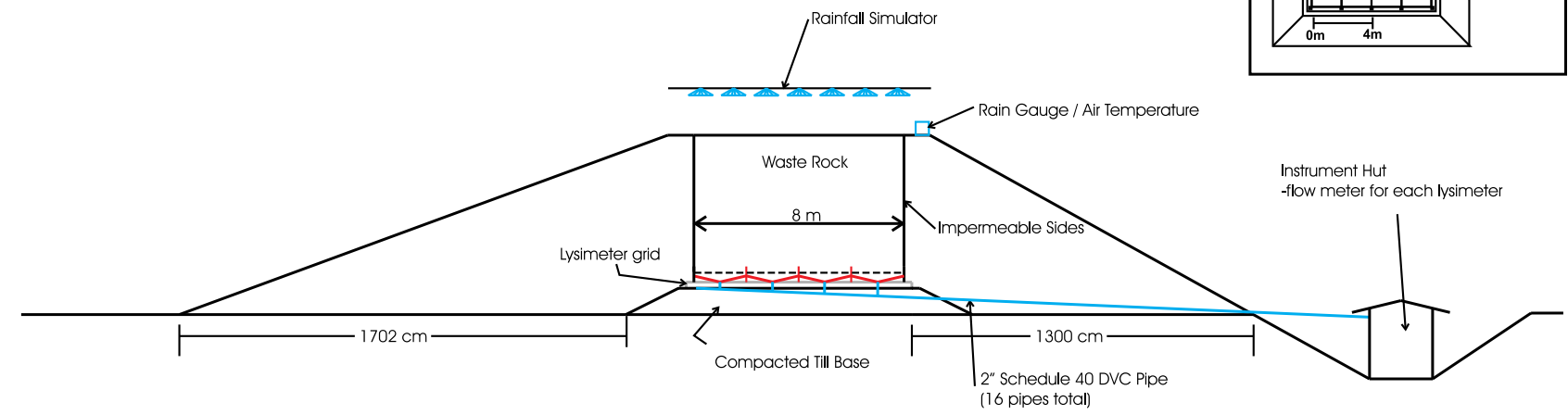

Figure 1. Simplified cross section of constructed pile experiment and plan view of experiment core (inset).

central core of the pile and prevent lateral diversion of flow around the zero pressure lysimeters at the base. The sides provided lateral no-flow boundaries, allowing all flow to be captured at the basal lysimeters but also limited the possible lateral extent of spatially distinct flow pathways. Waste rock was placed in the pile using a large excavator, taking care to prevent damage to instrumentation. The resulting waste rock texture matched the range of textures seen in excavations o existing waste rock piles on site. It was highly heterogeneQus, ranging from $1.0 \mathrm{~m}$ diameter boulders to clay, with
areas that were matrix supported and areas with matrix-free cobbles and boulders. No runoff was permitted and the soi surface was not vegetated. Outflow from each $2 \mathrm{~m}$ by $2 \mathrm{~m}$ lysimeter was separately piped to an instrumentation hut where

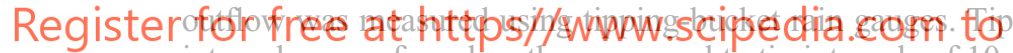
intervals range from less than a second to tip intervals of $10 \mathrm{~s}$ of minutes. The raw data set consists of the event times of each tip of the outilow meters. The raw tip data was converted to flow rate using meter-specific calibrations, erroneous data were manually removed, gaps filled, and then flow was interpolated to a $15 \mathrm{~min}$ time interval. A weather station with a rain gauge on top of the pile recorded incident natural and artificial rainfall events. Additional information about the setup can be obtained from Nichol et al. [2005].

\section{Modeling Approach}

\subsection{General Overview of the Methodology}

[12] The methodology is based on linear systems theory. The idea is to relate rainfall and outflow data in order to identify a nonparametric empirical transfer function. We then fit a model with two parameters to the empirical transfer function to provide a parameterized transfer function model.

[13] In linear systems, convolution is used to describe the relationship between three signals: the input signal $X$, the impulse response (i.e., the output of a delta function input signal) $f$, and the output signal $Y$. This relationship can be expressed as follows:

$$
Y(t)=\int_{-\infty}^{\infty} f(t-\tau) X(\tau) d \tau
$$

According to the spectral representation theorem, a stationary stochastic process $X$ has a spectral representation of the form

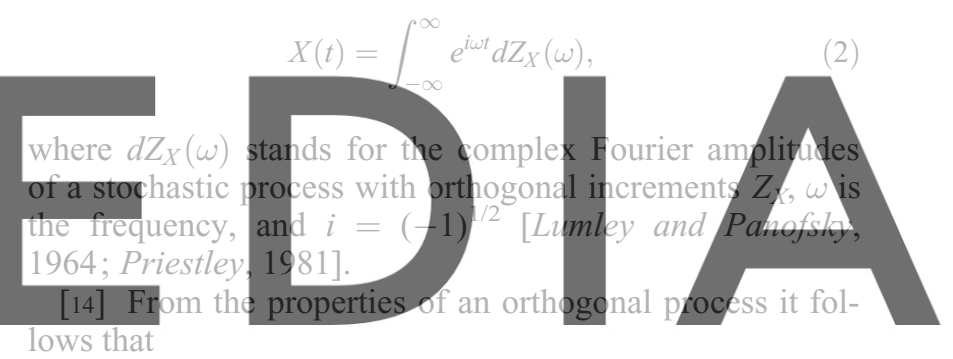

\section{download the kersion without whe watermark}

where $E[]$ is the expected value, the asterisk denotes the complex conjugate, $\phi_{X X}$ is the spectral density or spectrum of $X(t)$, and $\delta_{i j}$ is the Kronecker delta.

[15] Equations (2) and (3) allow expressing (1) in spectral representation

$$
\phi_{Y Y}(\omega)=|F(\omega)|^{2} \phi_{X X}(\omega),
$$

where $\phi_{Y Y}(\omega)$ is the spectrum of the output signal and $|F(\omega)|^{2}$ is the transfer function $(T F)$.

\subsection{Estimation of the $\boldsymbol{T F}$ From Outflow Data}

[16] The spectral signature of the water released at the bottom of the pile and recorded as outflow in each lysimeter is now analyzed. Data comes from an eight-month period (1 March to 31 October 2000) with fluxes of $10^{-8}$ to $10^{-6} \mathrm{~m} \mathrm{~s}^{-1}$. During the winter season the top of the pile is frozen and so precipitation does not produce input. Drainage decreases monotonically from November to March, following a similar drainage curve in each lysimeter to a flux of $<1 \times 10^{-9} \mathrm{~m} \mathrm{~s}^{-1}$. The winter part of the time series is not included in this study.

[17] The two variables compared are rainfall $r$ and outflow $q$. In each individual lysimeter the transfer function is 
given from (4) as the ratio between the spectrum of the output $\phi_{q q}(\omega)$ (i.e., outflow through a given lysimeter) divided by the spectrum of rainfall $\phi_{r r}(\omega)$ (the same for all lysimeters) and can be expressed as

$$
\left|F_{\text {emp }}(\omega)\right|^{2}=\frac{\phi_{q q}(\omega)}{\mu \phi_{r r}(\omega)},
$$

where $\left|F_{\text {emp }}(\omega)\right|^{2}$ is the empirical transfer function and $\mu$ is a normalization coefficient. This coefficient is incorporated in the model to account for the differences between the theoretical cumulative infiltration value within a given cross sectional area of the soil surface to the cumulative outflow measured through the projection of that cross sectional area to the outflow measurement depth. In our experiment this is the footprint of $2 \mathrm{~m} \times 2 \mathrm{~m}$ individual lysimeters, or larger areas covering several lysimeters at once.

\subsection{Data Analysis}

[18] We examine transfer functions for all 16 individual lysimeters at a first stage. Additionally, we aggregate the output signal to represent multiple lysimeters, considering the pile divided into quarters and globally as a single unit.

[19] The rainfall data for the study period consists of a time series of 3221 unevenly spaced tipping-bucket rain gauge event times. Data was then converted into a flow
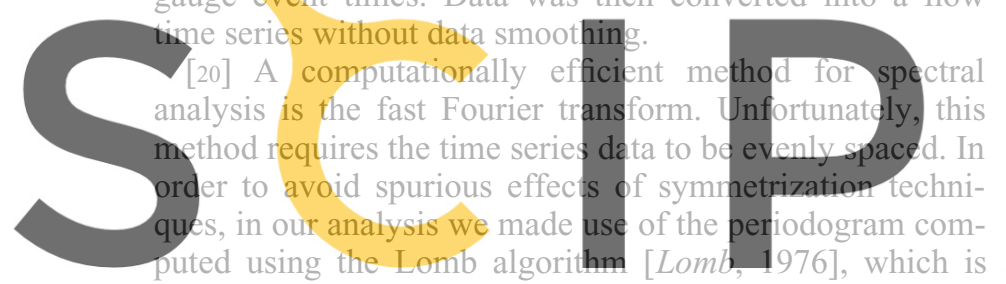

especially suited for unevenly spaced data. Also, a spline

Register cosine bell taper is applied, to minimize the influence of

[21] The periodogram of the rainfall is showed in Figure

2a. The periodogram has no obvious trend with respect to frequencies, indicating that rainfall can be considered a stationary stochastic process, with short time correlation. Figure $2 \mathrm{~b}$ shows the spectrum of the outflow through lysimeter 9

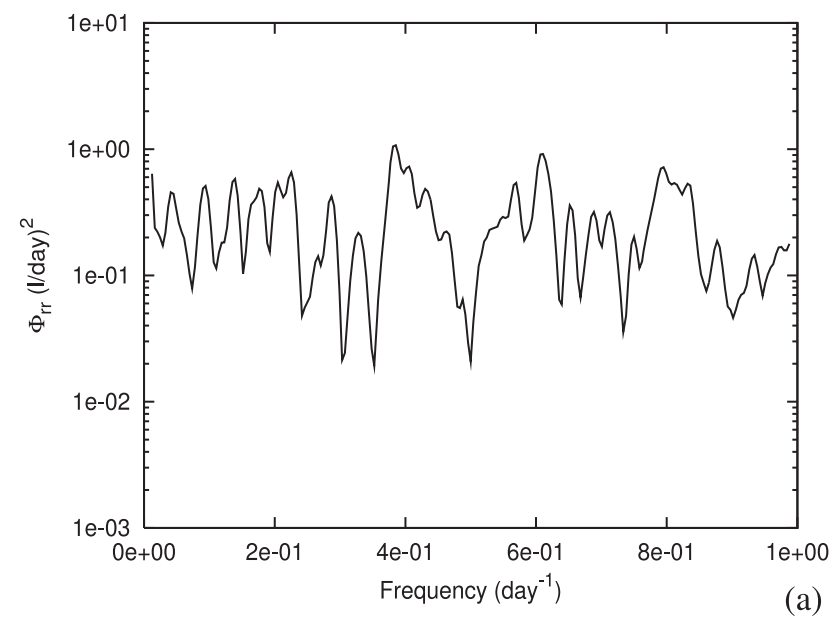

for comparison. This section of the pile is acting as a lowpass filter attenuating the input signal at high frequencies. The residual signal at high frequencies is produced by a fast flow component (i.e., preferential flow) that results in an asymptotic behavior of the spectrum. The relative noise of the spectrum (as well as all the other ones presented in this paper) is related in part to the acquisition system, based on tipping buckets rather than a continuous record.

\subsection{Conceptual Model}

[22] The two components of flow (i.e., fast and slow) have been decoupled assuming that the rate of flow is different in the two zones of the domain (i.e., matrix and macropores). In our model we assume that there is no interchange between the two zones and that the water that infiltrates through the macropores is released instantaneously (in a higher pile large delay could be expected and included in the model). Using spectral representation we can express the water outflow in the basal lysimeters as follows:

$$
d Z_{q}(\omega)=\left[F_{\text {matrix }}(\omega)(1-\alpha)+\alpha\right] d Z_{r}(\omega)
$$

where $d Z_{q}$ and $d Z_{r}$ are the spectral amplitudes of the outflow and the effective infiltration, respectively, $\alpha$ is the fraction of the effective infiltration that is driven through the macropores, and $F_{\text {matrix }}$ is the spectral amplitude of the
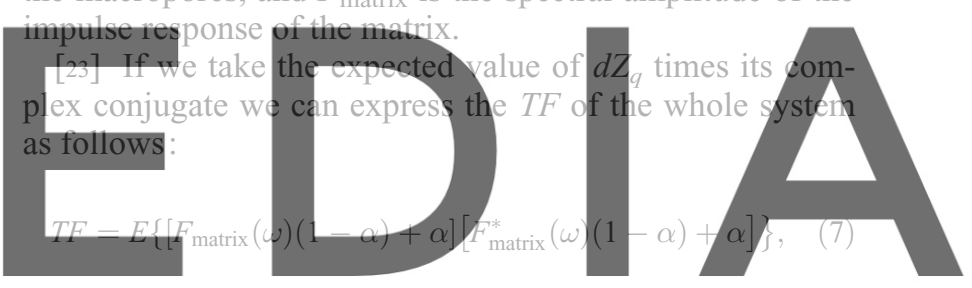

An explicit expression of (7) is obtained by considering

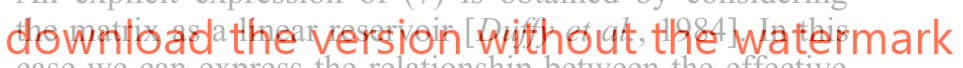
case we can express the relationship between the effective infiltration and the outflow through the matrix as follows:

$$
\frac{d V(t)}{d t}=r_{\text {matrix }}(t)-q_{\text {matrix }}(t)
$$

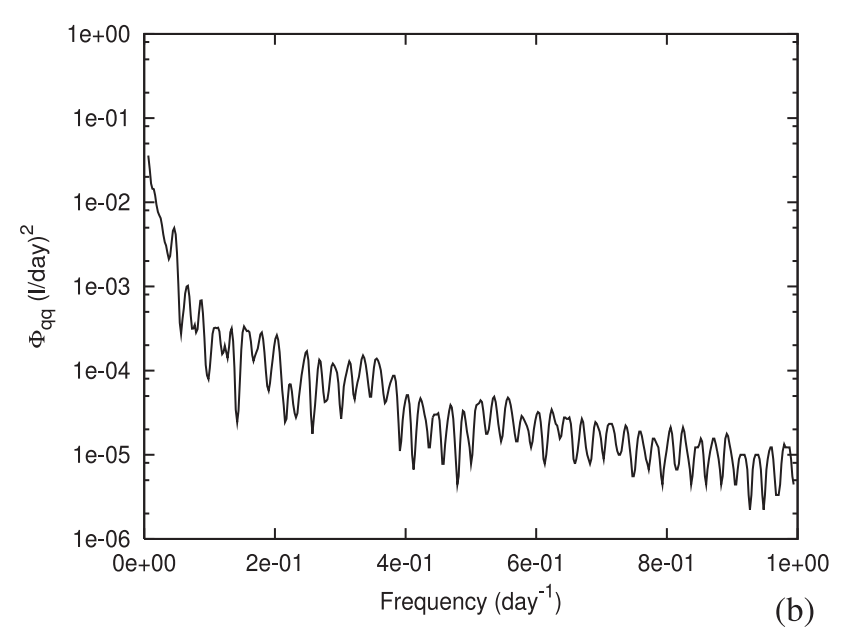

Figure 2. Periodograms of (a) rainfall and (b) the outflow through lysimeter 9. 
where $r_{\text {matrix }}=(1-\alpha) r$ and $q_{\text {matrix }}$ are the effective infiltration and the outflow through the matrix, and $V$ is the volumetric soil moisture per unit area given by

$$
V(t)=\int_{0}^{z_{0}} \theta(z, t) d z
$$

where $\theta$ is the local soil moisture content taking only the matrix into account, and $z_{0}$ is the height of the rock pile. The linear model adopted implies that the outflow $q_{\text {matrix }}$ is related to $V$ by

$$
q_{\text {matrix }}(t)=a_{l}\left[V(t)-V_{0}\right],
$$

where $a_{l}$ is the soil-moisture reservoir coefficient and $V_{0}$ is the minimum soil-moisture storage approximately equal to residual moisture content (for example, measured at the end of the winter period) times the vertical extent of the unsaturated zone $\left(V_{0}=\theta_{0} z_{0}\right)$.

[24] In the linear model, water infiltrating at surface will immediately begin to raise the total water content $[V(t)]$ (equation (9)) leading to an immediate change in outflow (equation (10)), that will increase linearly with precipitation. We recognize that a matrix wetting front would require a finite time to reach the base of the pile for an outflow response to occur. This means that some outflow volume is attributed to outfloy

hort times, and hence some macropore flow $(\alpha$, equation

(6)) may be underestimated. This duality has importan implications in upscaling and model predictability to be

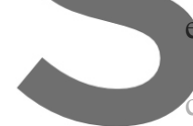

xplored later. cient $\left(a_{l}\right)$ has dimensions of inverse time units $\left(\left[T^{-1}\right]\right)$. In coarse portion of the pile, with steep soil water and hydraulic conductivity characteristic curves, a small change in

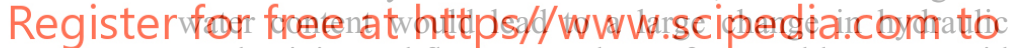
conductivity and flux rate at the surface, and hence a rapid movement of a wetting front. In the linear model, a large $a_{l}$ vaiue in (10) leads to cioser match of input and output fiow rate (9), and hence a lower overall change in storage over the event. In this way, $1 / a_{l}$ can be somewhat related to a characteristic time that is representative of the lag time of the water released by the matrix. It is not equal to a propagation velocity of a wetting front, but quantifies the time scale of dissipation of a perturbation taking place at the surface of an equivalent matrix reservoir.

[26] By combining (8) and (10) and using spectral representation we can write $F_{\text {matrix }}$ as

$$
F_{\text {matrix }}=\frac{1-i \omega / a_{l}}{1+\omega^{2} / a_{l}^{2}}
$$

By substituting $F_{\text {matrix }}$ and its complex conjugate into (7) we obtain an explicit expression of the $T F$ of the system

$$
T F(\omega)=\frac{1}{1+\Omega^{2}}\left(1-\alpha^{2}\right)+\alpha^{2},
$$

where $\Omega=\omega / a_{l}$. From Figure 3 we see that when there is no preferential flow $(\alpha=0) T F$ decreases as a power law in the logarithm of the dimensionless frequency $\Omega$ (actually $\Omega^{-2}$ ). As already observed in section 3.3, in the presence of

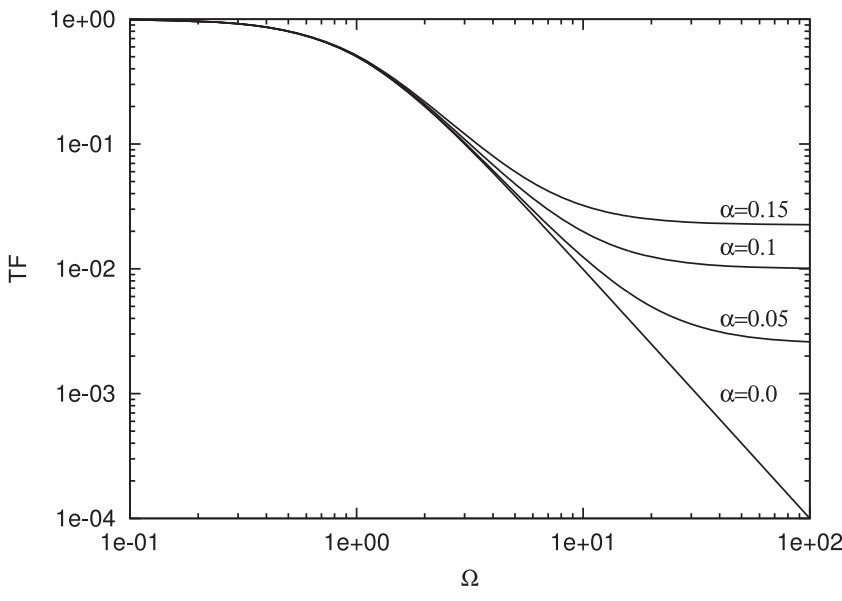

Figure 3. Dimensionless parametric $T F$ as a function of different $\alpha$ values.

preferential flow, the transfer function shows an asymptotic behavior where the impulse response of the signal at high frequencies is proportional to $\alpha^{2}$.

\section{Calibration, Model Performance, and}

\section{Discussion}

\subsection{Individual Lysimeters
[27] First, we analyze the $\mu$ coef
before, this coefficient relates the outflo
inflow at the arda located at the top of t
ing to the soil surface projection of the fo
measurement area at depth.}

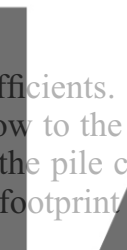

[28] Thus, in our experimental setup, it accounts mainly for horizontal water redistribution within the different secd awnbo adp thenversionsibsilithaket the lataterfmark heterogeneity of the medium. Information about this spatial dependency can be obtained a priori by analyzing the amount of water released by each lysimeter (Table 1). While the average flow recorded in the system is $0.82 \mathrm{~m}^{3}$ per lysimeter in the considered period, actual registered

Table 1. Summary of the Water Released by Each Lysimeter During the Period Considered

\begin{tabular}{cc}
\hline Lysimeter Number & Water Released $\left(\mathrm{m}^{3}\right)$ \\
\hline 1 & 0.68 \\
2 & 0.77 \\
3 & 0.39 \\
4 & 0.62 \\
5 & 0.44 \\
6 & 2.01 \\
7 & 0.82 \\
8 & 0.57 \\
9 & 0.56 \\
10 & 0.86 \\
11 & 0.89 \\
12 & 0.71 \\
13 & 1.37 \\
14 & 0.82 \\
16 & 0.80 \\
Average & 0.75 \\
& 0.82 \\
\hline
\end{tabular}


outflow values range from $2.01 \mathrm{~m}^{3}$ in lysimeter $6(244 \%$ of the mean value) to $0.39 \mathrm{~m}^{3}$ for lysimeter $3(47 \%)$. Notice that in a real pile the infiltrated amount would not be equal to rainfall due to the combination of runoff and evaporation processes at the top of the pile. These effects could either be filtered a priori (performing a water balance at the soil surface), or included within $\mu$. It is worthwhile to note that the individual outputs are highly heterogeneous. This is illustrated for example by the analysis of the outflow in lysimeters 8 and 9 (Figure 4). Analyzing the responses to the individual artificially induced precipitation event of 18 July 2000, the outflow from lysimeter 8 shows a fast and sharp response, while that of lysimeter 9 is smooth and slow (Figure 4b). Thus, although both lysimeters released almost the same total amount of water during the full nine-month period (i.e., similar $\mu$ value), their spectral density functions are very different (Figure 4a), in particular when looking at the output signal at high frequencies (i.c., short times).

[29] Next, each empirical $T F$ has been parameterized using equation (12). The two fitting parameters $\left(a_{l}\right.$ and $\left.\alpha\right)$ have been determined by a trial and error approach that minimizes the sum of squared differences between the empirical $T F$ and the parametric $T F$. The results of the fitting are shown in Figure 5 and summarized in Table 2 for each lysimeter with the exception of lysimeters 10,11 , and 12 that have a noisy time series that makes the calibration unreliable (Figure 6).

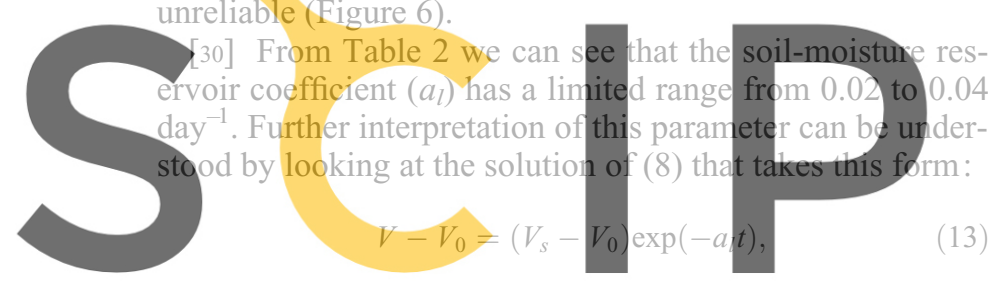

where $V_{S}$ is the initial soil-moisture content averaged over

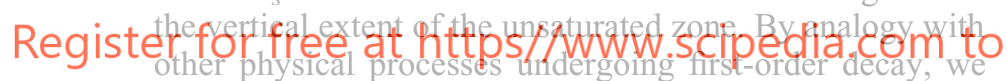
can define a characteristic time of water remaining in the matrix in the following form:

$$
t_{c}=\frac{\ln (2)}{a_{l}} .
$$

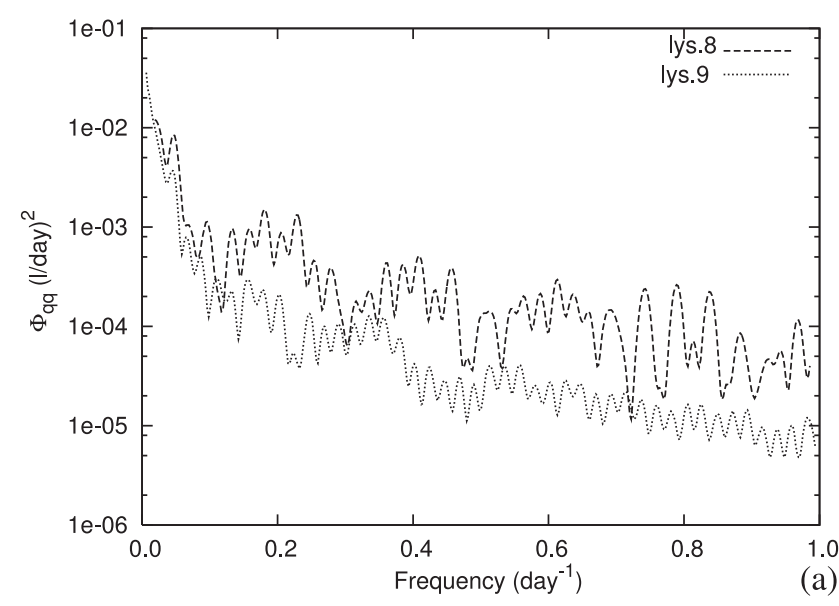

Here $t_{c}$ represents the time when half of the volume of the recharge impulse occurring at the top and not flowing through macropores has reached the bottom. The characteristic times obtained from the calibration range between 17 and 34 days. This is averaged over all events of varying duration and starting water content $\left(V_{s}\right)$ within the eightmonth period. For a $5 \mathrm{~m}$ pile, this means average characteristic velocities ranging from 0.14 to $0.29 \mathrm{~m} \mathrm{~d}^{-1}$.

[31] It is worthwhile to note that in the calibration process, $a_{l}$ values are strongly influenced by the fastest components of the discharge through the matrix while the very slow base discharge is implicitly neglected because of the time series that we analyze is only eight months in duration. The total infiltrated amount leads to an average infiltration of $0.31 \mathrm{~m} \mathrm{yr}^{-1}$. An additional experiment performed at the site included a tracer test running from September 1999 to August 2002. The first year of data determined that cumulative tracer recovery per lysimeter ranged from 11\% to $117 \%$ of the tracer mass applied above each lysimeter which encompasses both variations in total flow, and in macropore or preferential flow $(\alpha)$ [Nichol, 2002; Nichol et al., 2005]. From the measured transport data preliminary estimates of median transport time for the whole pile ranges from 2.0 to 3.9 years, This reinforces that $a_{l}$ is related to average response time rather than actual residence time.

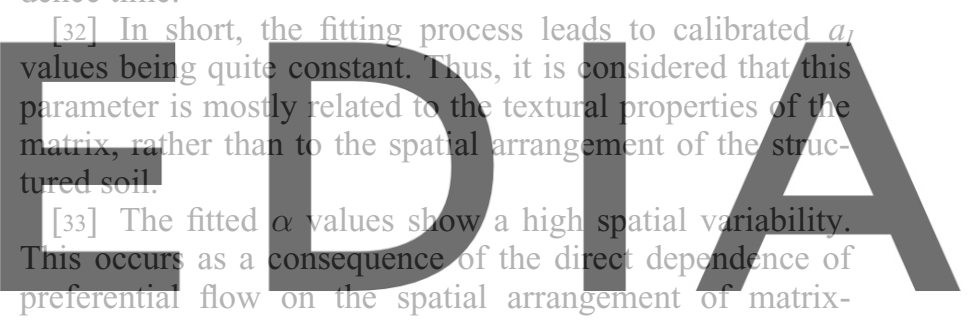
supported and matrix-free zones. In the study of Nichol

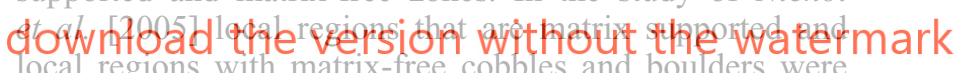
observed during construction. From Table 2 we see that there is a set of lysimeters that has a very low fraction of preferential flow (lysimeters 2, 3, 4, 13, 14, and 15). This fraction is lower than $1 \%$ out of the total flow volume but an accurate estimation of this value is not possible because

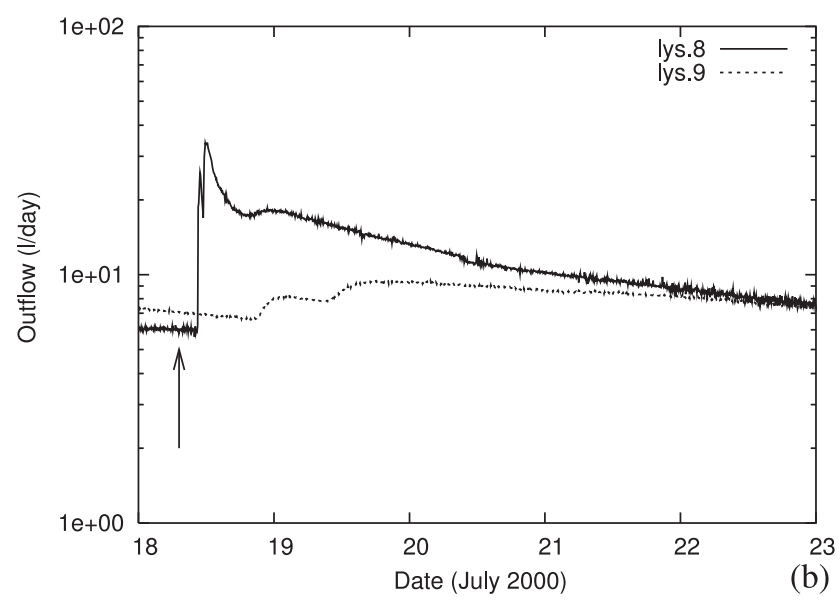

Figure 4. (a) Periodograms of outflow through lysimeters 8 and 9 compared to (b) actual outflow collected at the same lysimeters after the application of a single artificial rainfall event (indicated by the arrow). 


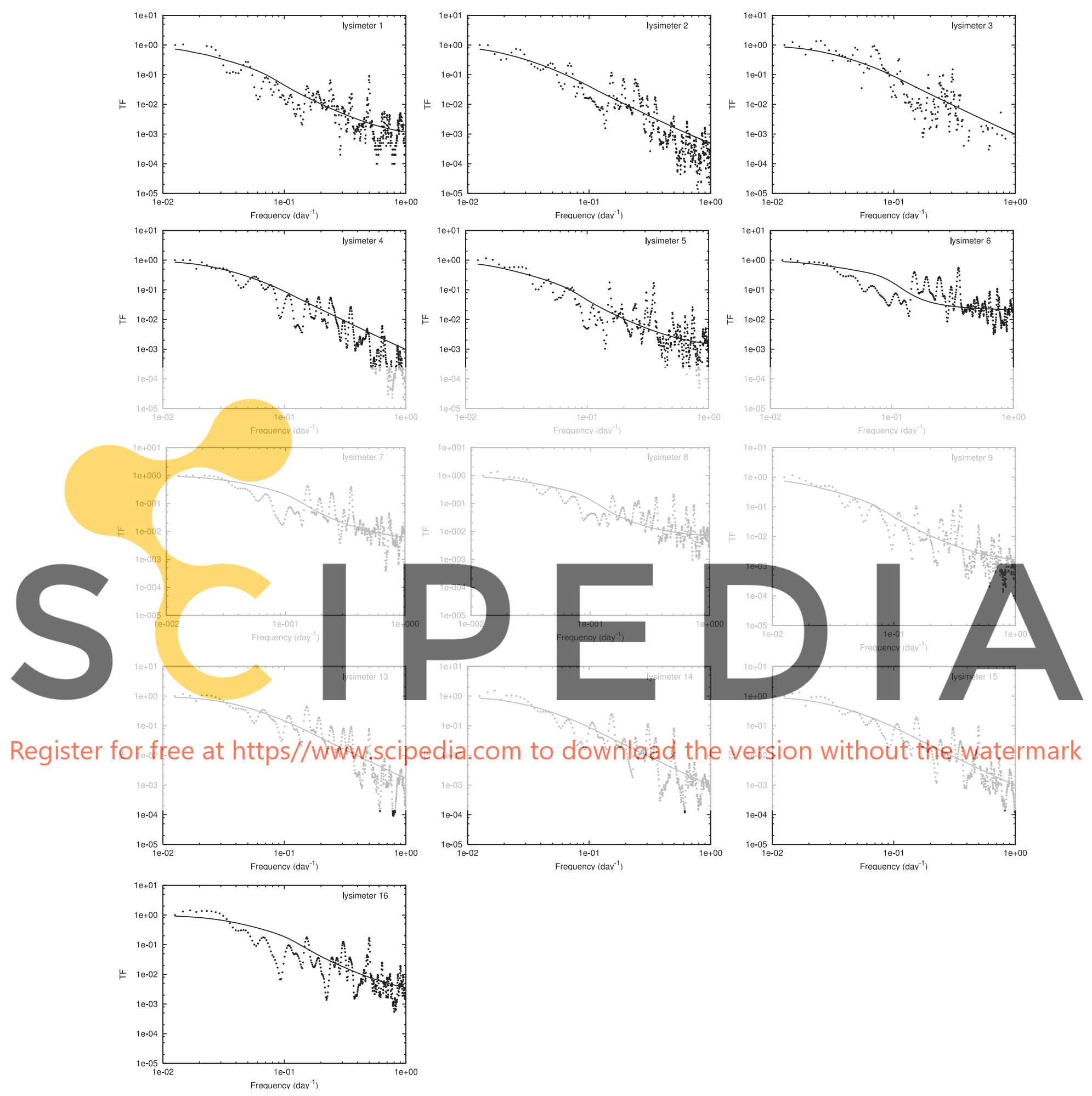

Figure 5. Empirical $T F$ (dotted line) and simulated $T F$ (solid line) for each lysimeter (except lysimeters 10,11 , and 12).

the signal of the corresponding outflow time series at high frequencies is very low. Another set of lysimeters (lysimeter $1,5,9$, and 16) shows a moderate rate of preferential flow ranging from $3 \%$ to $5 \%$ while the remaining lysimeters (lysimeter 6, 7, and 8) show an important component of preferential flow ranging from $9 \%$ to $15 \%$. It is interest- ing to note that the lysimeters belonging to each group are spatially clustered. This scale of clustering suggests heterogeneity at scales larger than the largest boulder size and may be related to the construction technique or the random position of the very large $(>0.5 \mathrm{~m}$ diameter $)$ boulders within the pile. On the other hand, the $\alpha$ value and the 
Table 2. Results of the Calibration for Each Individual Lysimeter

\begin{tabular}{ccc}
\hline Lysimeter & $a_{1}\left(\mathrm{~d}^{-1}\right)$ & $\alpha$ \\
\hline 1 & 0.02 & 0.03 \\
2 & 0.02 & $<0.01$ \\
3 & 0.03 & $<0.01$ \\
4 & 0.03 & $<0.01$ \\
5 & 0.02 & 0.03 \\
6 & 0.03 & 0.15 \\
7 & 0.04 & 0.07 \\
8 & 0.03 & 0.08 \\
9 & 0.02 & 0.03 \\
13 & 0.04 & $<0.01$ \\
14 & 0.03 & $<0.01$ \\
15 & 0.03 & $<0.01$ \\
16 & 0.04 & 0.05 \\
\hline
\end{tabular}

amount of water released by each individual lysimeter during the period considered (given by $\mu$ ) do not display a clear spatial correlation (see Figure 7).

\subsection{Lysimeters Organized Into Clusters}

[34] The same analysis that was carried out for individual lysimeters has also been performed on outflow data created by aggregating the outflow from contiguous lysimeters into quarters of the pile as indicated in Figure 8. The results of the calibration to these aggregated outflows are shown in Figure 9 and summarized in Table 3. Notice that in erder to produce the mean values we ters 10,11 , and 12 , where we cou ibration. It turns out that all qu

calibrated $\alpha$ value that is lower th
the $\alpha$ values of their individual ly
are taken as the proportion of the
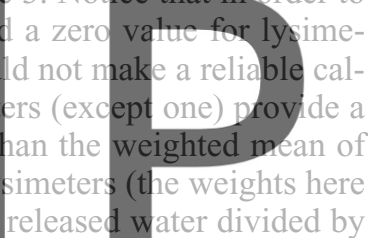
the total one). This observation is confirmed by a further upscaling obtained by aggregating all the lysimeters to-

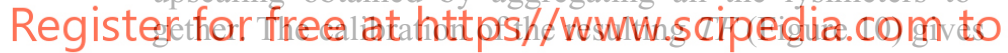
an estimated $\alpha$ equal to 0.02 indicating that at large scales the pile is mostly acting as an equivalent matrix reservoir, while the weighted average from the 16 small lysimeters is close to 0.04 .

[35] The somewhat surprising point is that the $\alpha$ values do not upscale arithmetically. The reason is associated with the slight heterogeneity in $a_{l}$, meaning that water does not reach the bottom of the pile at exactly the same times. The high frequency responses that would contribute to higher values of alpha in individual lysimeters combine together to form an averaged response at the pile level which has a lower spectral frequency. Thus, when looking at the

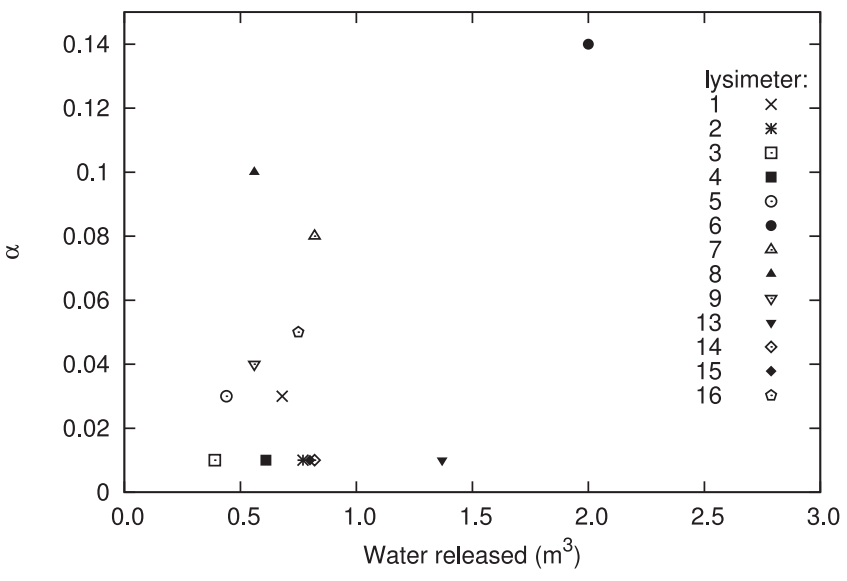

Figure 7. Water released during the period considered (1 March - 30 October 2000) versus calibrated $\alpha$.

upscaled lysimeter, some of the water really flowing through a macropore is considered as flowing through the matrix. This way, the actual separation between the two flow systems is scale dependent. This observation has a strong implication in the potential release of chemicals from the pile, associated with the different processes taking place in the macropores and the matrix

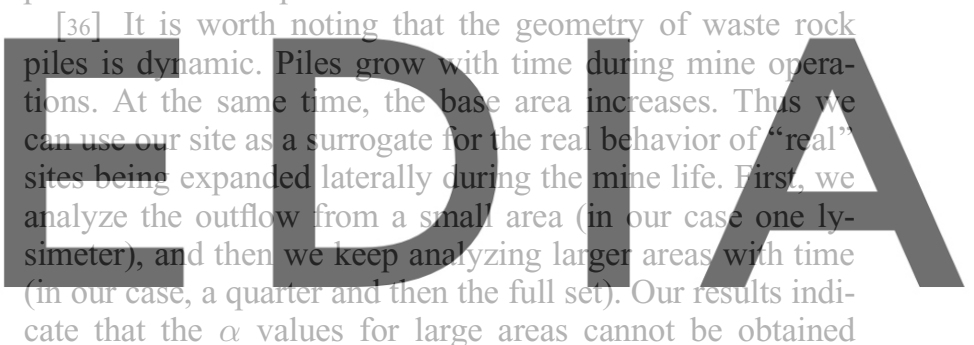

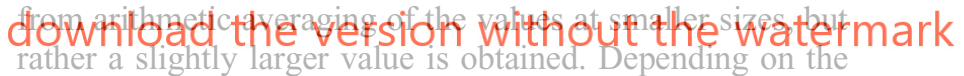
problem at hand this result is conservative or risky. For example, for leachate evaluation the safe option is using the smallest possible $\alpha$ values, and so arithmetic averaging will be on the safe side. Another interesting result is that $a_{l}$ is quite constant, and thus can be upscaled without problems.

\section{Conclusions}

[37] We modeled water flow through a highly heterogeneous unsaturated medium using a double porosity model, including macropores and the presence of a conductive matrix. The latter is modeled by a linear transfer function
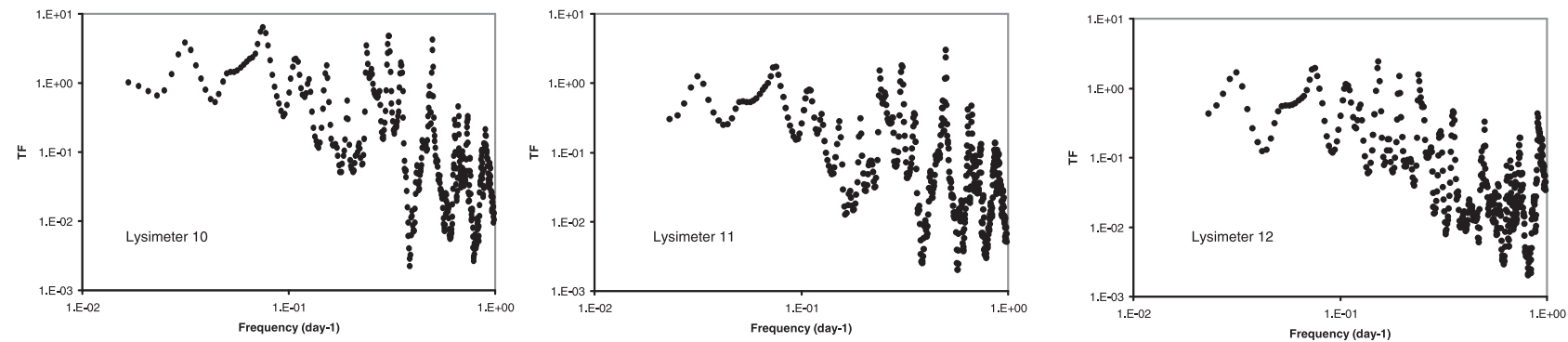

Figure 6. Empirical $T F$ for lysimeter 10, 11, and 12. The noise prevented obtaining a reliable calibration. 


\begin{tabular}{|c|c|c|c|}
\hline 4 & 8 & 12 & 16 \\
\hline \multicolumn{2}{|c|}{ NW } & \multicolumn{2}{|c|}{ NE } \\
\hline 3 & 7 & 11 & 15 \\
\hline 2 & 6 & 10 & 14 \\
\hline \multicolumn{2}{|c|}{ SW } & \multicolumn{2}{|c|}{ SE } \\
\hline 1 & 5 & 9 & 13 \\
\hline
\end{tabular}

Figure 8. Location of the lysimeters aggregated into quarters.

from the top to the bottom of the pile. It is worth noting that our work relies on a simplified approach that aims at capturing quantitative information about the behavior of a very complex medium using just two lumped parameters.
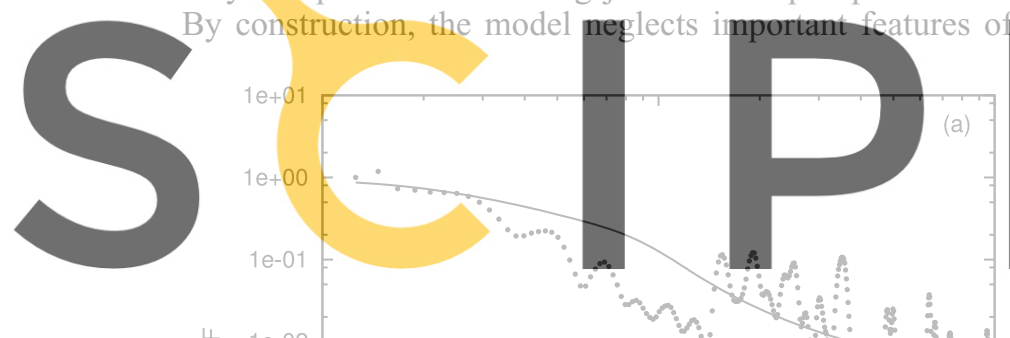

Register for free at https//www.scipediactom to
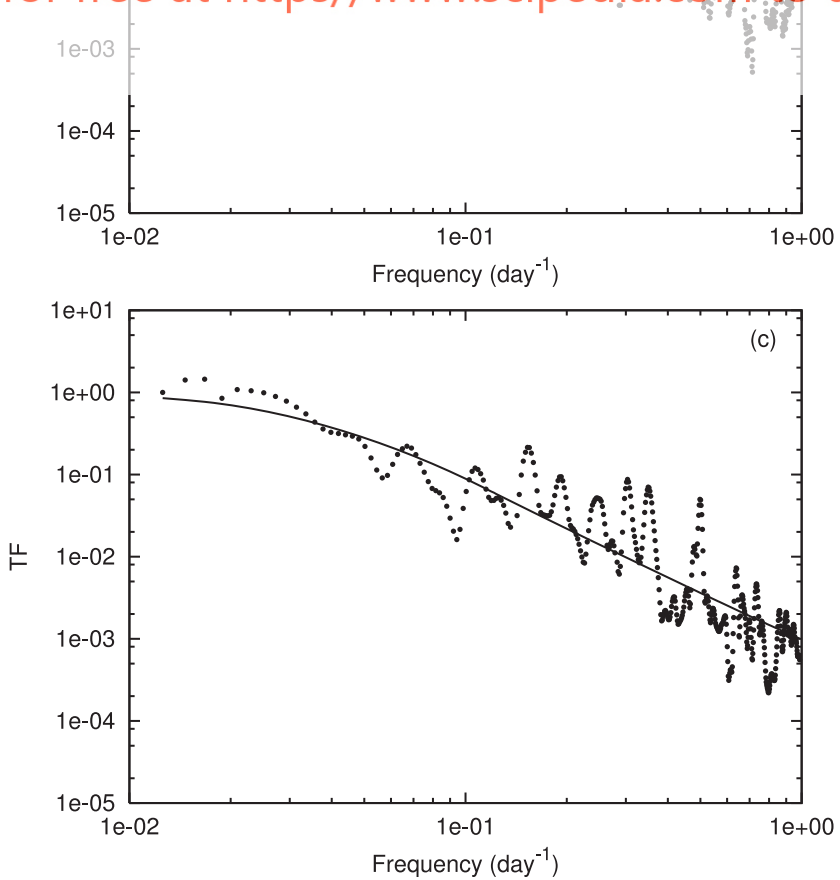

Table 3. Results of the Calibration for a Combination of Four Lysimeters Aggregated (Figure 8) and All Lysimeters Aggregated Together $^{\mathrm{a}}$

\begin{tabular}{lccc}
\hline \multicolumn{1}{c}{ Lysimeters } & $a_{1}\left(\mathrm{~d}^{-1}\right)$ & $\alpha$ & $\sum \beta_{i} \alpha_{i} / \sum \beta_{i}$ \\
\hline $\mathrm{SW}(1+2+5+6)$ & 0.02 & 0.07 & 0.09 \\
$\mathrm{NW}(3+4+7+8)$ & 0.03 & 0.02 & 0.04 \\
$\mathrm{SE}(9+10+13+14)$ & 0.03 & 0.02 & $0.01\left(^{*}\right)$ \\
$\mathrm{NE}(11+12+15+16)$ & 0.03 & 0.01 & $0.01\left(^{*}\right)$ \\
$\mathrm{SW}+\mathrm{NW}+\mathrm{SE}+\mathrm{NE}$ & 0.03 & 0.02 & 0.04 \\
\hline
\end{tabular}

${ }^{\mathrm{a}}$ The $\beta_{i}$ values are the amount of water released in each individual lysimeter and the sums go from $i=1$ to either 4 or $16 .\left(^{*}\right)$ indicates the average is performed with an incomplete data set.

unsaturated media, such as their hysteretic nature or the different spatial and temporal signature of preferential flow. Nonetheless, we argue that the main advantage of this methodology lies precisely in its simplicity. In fact, instead of using mechanistic models, which in these conditions pose problems in terms of reliability, the model is kept as simple as possible, while capturing the essential features of interest (i.e., the preferential flow fraction and some characteristic time of the flow through the matrix). The main conclusions of the study can be summarized as follows:

[38] 1. The variability in the amount of water released by each lysimeter highlights the important role played by horizontal redistribution. This effect is implicitly taken into

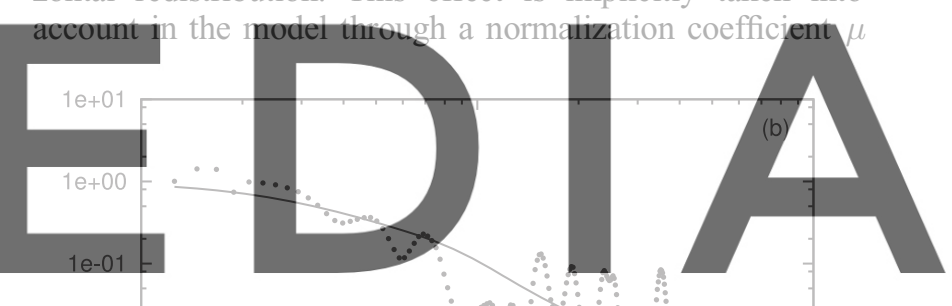

Figure 9. Empirical $T F$ (dotted line) and simulated $T F$ (solid line) for the lysimeters aggregated into quarters (a) SW, (b) NW, (c) SE, and (d) NE. 


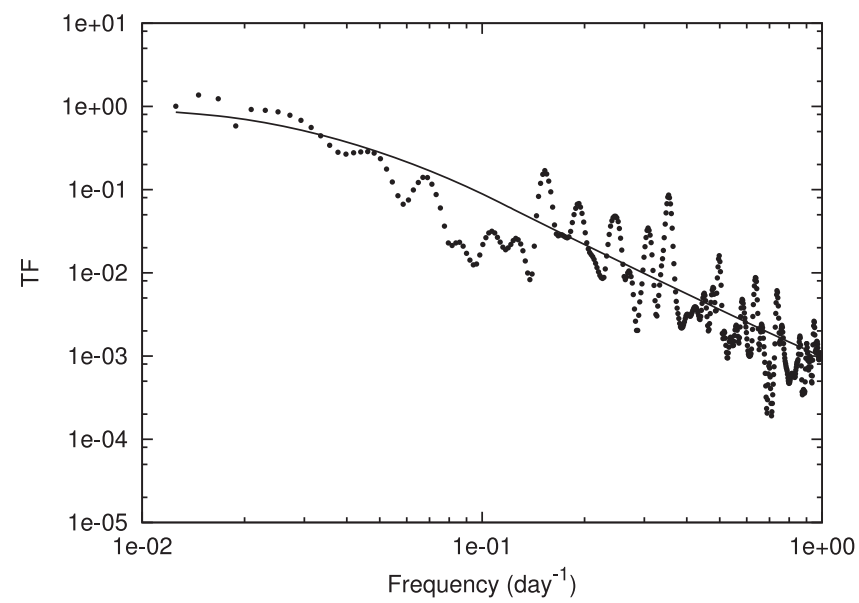

Figure 10. Empirical $T F$ (dotted line) and simulated $T F$ (solid line) when all lysimeters are aggregated into one.

(equation (5)). In a more general problem this same coefficient can also be used to include processes reducing infiltration through the pile, such as evapotranspiration or runoff.

[39] 2. The fitting of the parameteric transfer function model allows one to infer information about the characteristic time of the flow through the matrix (equation (14)). The results show that $a_{l}$ is quite constant, being related to the textural properties of the matrix and not to the spatial arrangements of the well-structured soil. The characteristic time inferred does not account for the flow components that travel slower than the eight-month period of data analyzed. 40] 3. The fitted $\alpha$ values display large spatial variability. different degrees of preferential flow observed within dependence of preferential flow patterns with textural heterogeneities occurring as a result of the construction procedure.

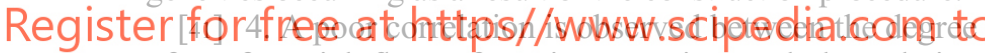
of preferential flow of a given section and the relative amount of water released by the same section.

[42] 5. If we repeat the calibration exercise by considering upscaled sections of the pile we see that the variability of $\alpha$ decreases and the behavior of the pile tends to approximate that of an equivalent matrix reservoir. The resulting upscaled $\alpha$ values are in general smaller than the predicted values that would be obtained by performing a weighted average of the small-scale values. This outcome would be especially important in determinations of actual leaching rates.

\section{Appendix A: Spectral Amplitude of the Impulse Response of the Matrix}

[43] Combining equations (8) and (10) one can express the relationship between the effective infiltration and the outflow through the matrix as follows:

$$
\frac{1}{a_{l}} \frac{d q_{\text {matrix }}(t)}{d t}+q_{\text {matrix }}(t)=r_{\text {matrix }}(t) .
$$

Equation (A1) can be rewritten using spectral representation

$$
\int_{-\infty}^{\infty} \exp (i \omega t)\left(\frac{1}{a_{l}} i \omega d Z_{q}+d Z_{q}-d Z_{r}\right)=0
$$

where $d Z_{q}$ and $d Z_{r}$ are the Fourier amplitudes of $q_{\text {matrix }}$ and $r_{\text {matrix }}$, respectively. It turns out that the only solution of (A2) is given by

$$
d Z_{q}=\left(\frac{1}{1+i \omega / a_{l}}\right) d Z_{r}
$$

From (A3) we can obtain the spectral amplitude of the impulse response of the matrix

$$
F_{\text {matrix }}=\frac{1-i \omega / a_{l}}{1+i \omega^{2} / a_{l}^{2}} .
$$

[44] Acknowledgments. PT acknowledges the financial support provided by the Torres Quevedo Programme of the Spanish Ministry of Science and Innovation (PTQ-09-01-00695). RDB acknowledges the support of the Programa Nacional de Avudas para la movilidad. Additional support by the Spanish Ministry of Science and Innovation (project ConsoliderIngenio, ref. CSD2009-00065) is gratefully acknowledged. The waste rock pile data was obtained from experiments that were conducted as part of the Waste Rock Hydrology Research Program, a project initiated between the University of British Columbia, the University of Saskatchewan, Cogema Resources Incorporated, Cameco Corporation, and the Natural Sciences and Engineering Research Council of Canada. The authors also want to thank Ruben Juanes and two anonymous reviewers for their very helpful comments.
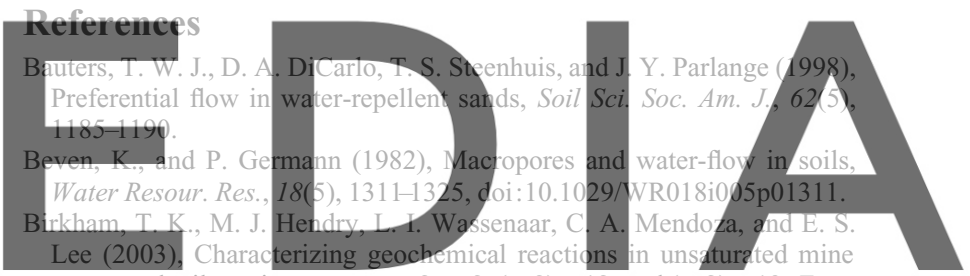

waste-rock piles using gaseous 0-2, co2, (co2)-c-12, and (co2)-c-13, Environ. Sci. Technol., 37(3), 496-501.

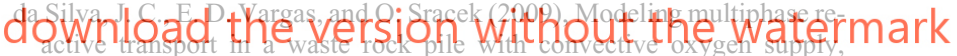 \\ Vadose Zone J., 8(4), 1038-1050}

Decker, D. L., and S. W. Tyler (1999), Evaluation of flow and solute transport parameters for heap leach recovery materiais, J. Environ. Quality, 28(2), 543-555.

Dekker, L. W., and C. J. Ritsema (1996), Preferential flow paths in a water repellent clay soil with grass cover, Water Resour. Res., 32(5), 12391249, doi: 10.1029/96WR00267.

Denic-Jukic, V., and D. Jukic (2003), Composite transfer functions for karst aquifers, J. Hydrol., 274(1-4), 80-94.

Dreiss, S. J. (1983), Linear unit-response functions as indicators of recharge areas for large karst springs, J. Hydrol., 61(1-3), 31-44.

Duffy, C. J., L. W. Gelhar, and P. J. Wierenga (1984), Stochastic-models in agricultural watersheds, J. Hydrol., 69(1-4), 145-162.

Edwards, W. M., M. J. Shipitalo, L. B. Owens, and L. D. Norton (1989), Water and nitrate movement in earthworm burrows within long-term notill cornfields, J. Soil Water Conserv., 44(3), 240-243.

Eriksson, N., A. Gupta, and G. Destouni (1997), Comparative analysis of laboratory and field tracer tests for investigating preferential flow and transport in mining waste rock, J. Hydrol., 194(1-4), 143-163.

Gerke, H. H. (2006), Preferential flow descriptions for structured soils, J. Plant Nutrition Soil Sci., 169(3), 382-400.

Gerke, H., and M. van Genuchten (1993), A dual-porosity model for simulating the preferential movement of water and solutes in structured porous media, Water Resour. Res., 29(1), 305-319, doi:10.1029/92WR02339.

Gish, T. J., and W. A. Jury (1983), Effect of plant-roots and root channels on solute transport, Trans. ASAE, 26(2), 440.

Hill, D. E., and J. Y. Parlange (1972), Wetting front instability in layered soils, Soil Sci. Soc. Am. Proc., 36(5), 697.

Jarvis, N. J. (2007), A review of non-equilibrium water flow and solute transport in soil macropores: Principles, controlling factors and consequences for water quality, Eur. J. Soil Sci., 58(3), 523-546. 
Jury, W. (1982), Simulation of solute transport using a transfer function model, Water Resour. Res., 18(2), 363-368, doi:10.1029/WR018i002 p00363.

Jury, W. (1999), Present directions and future research in vadose zone hydrology, in Vadose Zone Hydrology: Cutting Across Disciplines, edited by M. B. Parlange and J. W. Hopmans, pp. 432-442, Oxford Univ. Press, New York.

Jury, W., G. Sposito, and R. White (1986), A transfer function model of solute transport through soil 1. Fundamental concepts, Water Resour. Res., 22(2), 243-247, doi:10.1029/WR022i002p00243.

Jury, W., W. Gardner, and W. Gardner (1991), Soil Physics, 5th ed., John Wiley \& Sons, New York

Kabwe, L. K., G. W. Wilson, and M. J. Hendry (2005), Effects of rainfall events on waste-rock surface-water conditions and co2 effluxes across the surfaces of two waste-rock piles, J. Environ. Eng. Sci., 4(6), 469-480.

Kung, K. (1990a), Preferential flow in a sandy vadose zone: 1. Field observation, Geoderma, 46, 51-58.

Kung, K. (1990b), Preferential flow in a sandy vadose zone: 2. Mechanism and implications, Geoderma, 46, 59-71.

Lomb, N. R. (1976), Least-squares frequency-analysis of unequally spaced data, Astrophys. Space Sci., 39(2), 447-462.

Long, A. J., and R. G. Derickson (1999), Linear systems analysis in a karst aquifer, J. Hydrol., 219(3-4), 206-217.

Lopez, D., L. Smith, and R. Beckie (1997), Modeling water flow in waste rock piles using kinematic wave theory, in Proceedings of the Fourth International Conference on Acid Rock Drainage, pp. 497-513, Nat. Resour. Canada, Ottawa.

Lumley, J., and H. Panofsky (1964), The structure of atmospheric turbulence, Wiley-Interscience, New York.

Molenat, J., P. Davy, C. Gascuel-Odoux, and P. Durand (1999), Study of three subsurface hydrologic systems based on spectral and cross-spectral analysis of time series, J. Hydrol., 222(1-4), 152-164.

Molson, J. W., O. Fala, M. Aubertin, and B. Bussiere (2005), Numerical simulations of pyrite oxidation and acid mine drainage in unsaturated waste rock piles, J. Contaminant Hydrol., 78(4), 343-371.

Nichol, C. (2002), Transient flow and transport in unsaturated heterogeneous media: Field experiments in mine waste rock, Ph.D. dissertation, University of British Columbia, Vancouver.

Nichol, C., L. Smith, and R. Beckie (2005), Field-scale experiments of unsaturated flow and solute transport in a heterogeneous porous medium, Water Resour. Res., 41(5), W05018, doi:10.1029/2004WR003035.

Nicholl, M. J., R. J. Glass, and S. W. Wheatcraft (1994), Gravity-driven infiltration instability in initially dry nonhorizontal fractures, Water Resour. Res., 30(9), 2533-2546, doi:10.1029/94WR00164.

Nimmo, J. R. (2007), Simple predictions of maximum transport rate in unsaturated soil and rock, Water Resour. Res., 43(5), W05426, doi:10.1029/2006WR005372.

Philip, J. (1958), The theory of infiltration: 6 Effect of water depth over soil, Soil Sci., 85(5), 278.
Poisson, J., M. Chouteau, M. Aubertin, and D. Campos (2009), Geophysical experiments to image the shallow internal structure and the moisture distribution of a mine waste rock pile, J. Appl. Geophys., 67(2), 179-192.

Press, W., et al. (1986), Numerical Recipes, Cambridge University Press, Cambridge.

Priestley, M. (1981), Spectral Analysis and Time Series, Volume 1, 890 pp., Academic, New York.

Rasmussen, T. C., R. H. Baldwin, J. F. Dowd, and A. G. Williams (2000), Tracer vs. pressure wave velocities through unsaturated saprolite, Soil Sci. Soc. Am. J., 64(1), 75-85.

Reiter, M. (2009), Fluid flow estimates in molybdenum mine rock piles using borehole temperature logs, Environ. Eng. Geosci., 15(3), 175-195.

Roth, K., W. A. Jury, H. Fluhler, and W. Attinger (1991), Transport of chloride through an unsaturated field soil, Water Resour. Res., 27(10), 25332541, doi:10.1029/91WR01771.

Simunek, J., N. J. Jarvis, M. T. van Genuchten, and A. Gardenas (2003), Review and comparison of models for describing non-equilibrium and preferential flow and transport in the vadose zone, J. Hydrol., 272(1-4), 14-35.

Smith, L., and R. Beckie (2003), Hydrologic and geochemical transport processes in mine waste rock, Environ. Aspects Mine Wastes, 31, 51-72.

Smith, R. (1981), Rational models of infiltration hydrodynamics, in RainfallRunoff Relationship, edited by V. P. Singh, pp. 107-126, Water Resources Publications, Littleton, Colo.

Sracek, O., M. Choquette, P. Gelinas, R. Lefebvre, and R. V. Nicholson (2004), Geochemical characterization of acid mine drainage from a waste rock pile, mine Doyon, Quebec, Canada, J. Contaminant Hydrol., 69(1-2), 45-71.

van Genuchten, M., and P. Wierenga (1976), Mass transfer studies in sorbing porous media I. Analytical solutions, Soil Sci. Soc. Am. J., 40(4), 473.

Webb, G., S. W. Tyler, J. Collord, D. Van Zyl, T. Halihan, J. Turrentine, and T. Fenstemaker (2008), Field-scale analysis of flow mechanisms in highly heterogeneous mining media, Vadose Zone J., 7(3), 899-908.

White, R. E., J. S. Dyson, R. A. Haigh, W. A. Jury, and G. Sposito (1986), A transfer-function model of solute transport through soil. 2. Illustrative applications, Water Resour. Res., 22(2), 248-254, doi:10.1029/WR022 i002p00248.

Yamada, T., and M. Kobayashi (1988), Kinematic wave characteristics and new equations of unsaturated infiltration, J. Hydrol., 102(1-4), 257-266.

R. Beckie, Department of Earth and Ocean Sciences, University of British Columbia, Vancouver, BC V6T 1Z4, Canada.

C. Nichol, Department of Earth and Environmental Sciences, University of British Columbia Okanagan, Kelowna, BC V1V 1V7, Canada.

$X$. Sanchez-Vila, Department of Geotechnical Engineering and Geosciences, Universitat Politecnica de Catalunya BarcelonaTech, E-08034 Barcelona, Spain.

P. Trinchero, AMPHOS 21 Consulting S. L., Passeig de Garcia i Faria, 49-51, 1-1, E-08019 Barcelona, Spain. (paolo.trinchero@gmail.com) 